\title{
Anticipating policy considerations for a future HIV vaccine: a preliminary study
}

Emmanuel Ato Williams ${ }^{\mathrm{a}, \mathrm{b}}$, David J. M. Lewis ${ }^{\mathrm{c}}$, Sylvie Bertholet ${ }^{\mathrm{d}, \mathrm{e}}$, Maurizzio Zazzi ${ }^{\mathrm{f}}$

${ }^{a}$ Master Fellow in Vaccinology and Pharmaceutical Clinical Development, University of Siena, Italy

${ }^{\mathrm{b}}$ Core Medical Fellow, University Hospitals Southampton NHS Foundation Trust, Southampton General Hospital, UK

${ }^{\mathrm{c}}$ Professor of Clinical Vaccine Immunology, NIHR/Wellcome Trust Imperial Clinical Research Centre, Imperial College NHS Trust, UK

${ }^{\mathrm{d}}$ Head, T-cell platform, Translational Medicine, GlaxoSmithKline Vaccines S.r.l., Siena, Italy

${ }^{\text {e }}$ Scientific Director, PhD Program, GlaxoSmithKline Vaccines S.r.l., Siena, Italy

${ }^{\mathrm{f}}$ Professor of Clinical Microbiology, University of Siena, Siena, Italy 


\section{Anticipating policy considerations for a future HIV vaccine: a preliminary study}

Emmanuel Ato Williams ${ }^{\mathrm{a}, \mathrm{b}}$, David J. M. Lewis ${ }^{\mathrm{c}}$, Sylvie Bertholet ${ }^{\mathrm{d}, \mathrm{e}}$, Maurizzio Zazzi ${ }^{\mathrm{f}}$

${ }^{a}$ Master Fellow in Vaccinology and Pharmaceutical Clinical Development, University of Siena, Italy

${ }^{\mathrm{b}}$ Core Medical Fellow, University Hospitals Southampton NHS Foundation Trust, Southampton General Hospital, UK

${ }^{\mathrm{c}}$ Professor of Clinical Vaccine Immunology, NIHR/Wellcome Trust Imperial Clinical Research Centre, Imperial College NHS Trust, UK

${ }^{\mathrm{d}}$ Head, T-cell platform, Translational Medicine, GlaxoSmithKline Vaccines S.r.l., Siena, Italy

e Scientific Director, PhD Program, GlaxoSmithKline Vaccines S.r.l., Siena, Italy

${ }^{\mathrm{f}}$ Professor of Clinical Microbiology, University of Siena, Siena, Italy

\section{ABSTRACT \\ Background}

New Human Immunodeficiency Virus (HIV) infections continue to occur worldwide. Despite previous failures, there is renewed optimism about developing an effective HIV prophylactic vaccine following the $31.2 \%$ vaccine efficacy (modified intention to treat analysis) achieved in the RV-144 trial. Intense efforts at characterizing the immune responses in the trial participants who appeared to gain some protection from the candidate vaccine are ongoing to delineate correlates of protection. However, the characteristics of a vaccine suitable for programmatic introduction in high prevalence areas remains undefined.

\section{Aims}

We set out to ascertain the vaccination policies and strategies that policy makers involved in vaccine introductions would advise were a candidate HIV vaccine to become available.

\section{Methods}

Structured questionnaires in both English and French were self-administered to consenting policy makers such as members of National Immunization Technical Advisory Groups. Members from three out of the six WHO regional groups were purposively reached for their responses.

\section{Results}

Thirty-seven key opinion leaders were approached through self-administered questionnaires delivered by email or in person. Nine responses were received, representing a $24.3 \%$ response rate. The responses received were from 3 [Africa (6), Americas (1) and Europe (2)] out of the 6 WHO regions. All respondents would prioritize the vaccination of commercial sex workers over other risk groups if there was an effective HIV vaccine. Vaccine efficacy was considered to be the most important factor, ahead of vaccine safety and cost, in determining the acceptability of a new prophylactic HIV vaccine.

\section{Conclusions}

It is expected that the first generation HIV vaccines may be modestly efficacious. However, even a modestly efficacious vaccine might curtail the spread of HIV if universal or near-universal coverage is achieved. It is important to anticipate policy discussions which would influence how rapidly an HIV vaccine would be rolled-out programmatically to achieve maximum impact.

\section{INTRODUCTION}

A successful, safe and effective HIV vaccine may: 
a. Elicit neutralizing [1-3] and non-neutralizing [4, 5] antibodies against, as yet, not completely defined protective epitopes in order to be capable of providing protective immunity by decreasing susceptibility of vaccinees to persistent infection, and/or;

b. Stimulate a robust cell-mediated immune response capable of controlling replication after virus acquisition in order to reduce infectiousness and delay disease progression to AIDS [6].

c. Likely require priming vaccinations together with subsequent boosting vaccination(s), both with improved/novel adjuvants in order to achieve adequate immunogenicity [7].

d. Still be cost-effective and provide substantial public health impact provided sufficiently high or universal coverage levels, including populations at high risk, are attained $[8,9]$, even if of modest efficacy.

A partially effective vaccine might work by harnessing one or the other of the two arms of the immune system; more likely antibodies for a prophylactic vaccine, and the cell-mediated immune response for controlling an established infection. We do not know for sure yet whether an HIVspecific cell-mediated immunity may ever be effective at controlling an already established HIV infection, which may be impossible to eliminate. A fully effective vaccine may possess both a capacity to induce robust antibodies and cell-mediated immune response, as well as components of the innate immunity.

If such a vaccine becomes finally available, various modelling studies have shown the benefits and impact that could be derived under varying deployment and coverage levels $[8,9]$. These models are highly suggestive of the cost-effectiveness of the development of an HIV vaccine. The case of a successful HIV vaccine may well be very similar to that of the recently successful malaria vaccine that has been licensed [10]. However, no information exists concerning how countries and policy makers may decide which populations to vaccinate first when such a vaccine is finally available. 
We do not know which criteria may inform decisions on which programmatic strategies to employ in ensuring that an available vaccine is deployed to achieve maximum impact. It is possible that first generation HIV vaccines may be only modestly efficacious, as has been the experience with malaria vaccines. Additionally, it is anticipated that a successful HIV vaccine may be initially available in limited quantities [11]. Where constraints on availability are present, inexorably, there will be the question of prioritization that would best be answered pre-emptively.

This study was conducted to evaluate policy makers' views on such policy questions in an attempt to stimulate early discussions and foster country preparedness to readily introduce an effective HIV vaccine for maximum impact, especially in areas with high transmission or disease burden.

\section{METHODS}

The structured questionnaire [copy in supplementary material] designed for use in this study was written in English and translated into French. There were mostly closed-ended questions, but also a few open-ended questions, both aimed at eliciting opinions on policy considerations in the introduction of a new HIV-1 vaccine.

An introductory document gave respondents information about the study and background to the topic being reviewed. Both documents were administered together to targeted policy makers involved in new vaccine introductions, such as members of various National Immunization Technical Advisory Groups (NITAGs) (when applicable). A NITAG is a multidisciplinary group of national experts that provide independent, evidence-based advice to health authorities regarding vaccine policies, recommendations and introductions. The WHO has defined 6 basic criteria for a functional NITAG: a legislative/administrative basis, formal terms of reference, conflict of interests policy implemented, minimum of 5 main areas of expertise, meeting at least once a year, and a secretariat that distributes agenda and background material prior to meetings.

An effort was made to contact members (present or past) of well-established NITAGs in countries where these exist, such as the Joint Committee on Vaccines and Immunizations (JCVI) of the 
United Kingdom, and individuals who serve as key opinion leaders in new vaccine recommendations in countries where a formal NITAG is not existent (i.e. respondents from most African countries). In most African countries where a formal NITAG does not exist, we identified possible respondents involved in vaccine recommendations and/or distribution through the help of the African Regional Coordinator for Supporting Immunisation and Vaccines Advisory Committee Initiative. The other WHO regions: Eastern Mediterranean, Western Pacific and South East Asia could not be contacted.

Written informed consent was obtained from respondents before self-administration of the questionnaires. Suitable participants were purposively identified and targeted for contacting by email or in person.

As part of questions asked, vaccine efficacy was classified into 3 types defined as follows: vaccine efficacy for reduced susceptibility in vaccinated persons, VEs = [a]; vaccine efficacy for reduced rate of infection and/or rate of progression in vaccinated infected persons, VEp $=[b]$; and vaccine efficacy for reduced infectiousness in vaccinated infected persons, VEi $=[c]$.

In other instances, questions from the questionnaire graded on a 5-point Likert-type scale were transformed into 'positive' (for ' 1 '= 'strongly agree' and ' 2 ' = 'agree'), ' 0 ' = 'indifferent', and 'negative' (for ' 4 ' = 'disagree' and ' 5 ' = 'strongly disagree'), and summed up to obtain the ranking of responses.

We employed a system of ranking, where the rankings 1 to 8 made by respondents were accorded numerical weights of 8 through to 1 , respectively, based on which risk groups would be prioritized. These weights were then multiplied by the corresponding number of respondents and summed to derive a final ranking of the 8 risk groups asked about in this study. Similarly, the rankings 1 to 4 made by respondents of the factors that would influence an HIV vaccine's acceptability were accorded numerical weights of 4 through to 1 , respectively; the weights being multiplied by the corresponding number of respondents and summed to obtain a final ranking. 


\section{RESULTS}

This study approached 37 key opinion leaders through self-administered questionnaires delivered by email or in person. Nine responses were received, representing a $24.3 \%$ response rate. The responses received were from 3 [Africa (6), Americas (1) and Europe (2)] out of the 6 WHO regions. The countries from which responses were obtained included: Kenya and United Kingdom (2 responses each), Brazil, Mali, South Sudan, Uganda, and Zambia (1 response each). UK, Brazil, and Kenya have NITAGs that meet all 6 WHO basic criteria for functional NITAGs. Responses were not received from some key opinion leaders contacted by email by the time of manuscript preparation. There were non-responders from all three WHO regions mentioned. Sociodemographic parameters were not collected. The participants were either past or present members of national groups involved in vaccine recommendations in their countries (or regions). The responses given were meant to be those of individual participants and not necessarily that of the groups they represent.

For an initial HIV vaccination strategy, about forty-three percent of participants would prefer to vaccinate 'everyone at risk' (i.e. universal vaccination), while half (50\%) would prefer vaccinating targeted risk groups. Only $7 \%$ of the key opinion leaders would prefer vaccinating individuals considered to be at the highest risk (depending on country-/region-specific HIV epidemiological profile) [Table 1].

Where 'targeted risk groups' was chosen as the preferred initial HIV vaccination strategy, overall, $100 \%$ of participants would place 'commercial sex workers' first or second in terms of priority. The following risk groups were reported in decreasing order of priority by participants for initial HIV vaccination:

Commercial sex workers (questionnaire did not specify whether male or female) $=23$; High-risk heterosexuals $=18$; Intravenous drug users $=15$; Males who have sex with males $=14$; Male STDinfected $=12$; Healthcare workers occupationally exposed to blood $=11$; Women of reproductive 
age, especially adolescent females $=11$ and Military conscripts $=4$. The values stated represent the outcomes of the system of ranking employed which has been described in the Methods section.

The participants also ranked the following 4 vaccine attributes they expect to influence an HIV vaccine acceptability in a decreasing order of importance: Vaccine efficacy $=32$; Vaccine safety $=$ 26; Vaccine cost $=19$ and Vaccine type $=13$. In other words, a potential HIV vaccine would be more or less likely acceptable to the study participants according to the following ranking: vaccine efficacy $>$ safety $>$ cost $>$ type [Table 2]. Again, the values stated represent the outcomes of the system of ranking employed which has been described in the Methods section.

In terms of specific definitions of vaccine efficacy, a successful vaccine without VEs but with VEp and VEi will be the most acceptable (66\%). Participants were more or less likely to accept other vaccine efficacy characteristics or cost-effectiveness as represented in Table 3.

Finally, if an HIV vaccine with $\geq 95.98 \%$ vaccine efficacy became available, fifty percent of the study participants would be willing to pay up to 100 United States Dollars (USD) for a full course of HIV vaccination out-of-pocket (if they did have to pay), whiles $13 \%$ each would be willing to pay up to 500 USD or 1000 USD for a full course. One out of every 4 participants was willing to pay 'any amount' out-of-pocket for a full course as long as the HIV vaccine had $\geq 95.98 \%$ vaccine efficacy [Table 1].

\section{DISCUSSION}

The results of this survey suggest that nearly 1 out of 2 policy makers would recommend vaccinating 'everyone at risk' (i.e. universal vaccination) or vaccinating targeted risk groups for an initial HIV vaccination strategy. Only $11 \%$ of the key opinion leaders would prefer vaccinating individuals considered to be at the highest risk (depending on country-/region-specific HIV epidemiological profile). A WHO-UNAIDS consultation group suggested that initial deployment of HIV vaccines could proceed through targeted campaigns [11] - one of the two strategies endorsed by nearly $50 \%$ of respondents in this study. The country-specific implementation strategy would 
depend on a number of factors including the country's programmatic objectives for HIV control (e.g. protection of individuals, prevention of disease, reduction of cost burden through antiretroviral therapy (ART), disease control, disease elimination or eradication), existing national structures, availability of funds, 'core' transmitters within the population and the 'mixing behaviour' of risk groups, and the possibility of phased introductions in different sections of the population. The distribution method best suited for an individual country could be assessed using mathematical models of HIV transmission for the population in question, taking into account the vaccine availability to the country and its distribution possibilities [11]. Sufficient quality data from further studies are required to meaningfully guide these prospects. Although HIV is a global problem and its transmission occurs both internationally and locally, nation-driven distribution strategies based on national experience with existing vaccination programs (such as through the Expanded Program on Immunization, or ad-hoc mass immunisation campaigns to control epidemics) as opposed to an internationally driven strategy, may be more decisive in the level of vaccination coverage achieved. Such local approaches will of course be guided and supported by the international community, which for instance, will set targets that nations and regions would need to meet. Subsequently, perhaps many years down the line, an international 'end game strategy' might then become necessary if we interrupt ongoing HIV transmissions and approach the cusps of global HIV eradication, as is the case for polio presently.

Within individual countries, universal rather than risk-targeted approaches to roll-out a future HIV vaccine has been advocated to help mitigate the stigma historically associated with HIV/AIDS, and to encourage HIV vaccination by reducing social barriers [12]. A universal approach is also advocated to help avoid the pitfalls found in the risk-targeted deployment of the hepatitis B vaccine [13].

In this study, where 'targeted risk groups' was chosen as the preferred initial HIV vaccination strategy, the policy makers ranked from highest to least priority to receive the vaccines: commercial sex workers, high-risk heterosexuals, intravenous drug users, and males who have sex with males in 
the top four. Healthcare workers occupationally exposed to blood were ranked $6^{\text {th }}$. To the best of our knowledge, this is the first time such a data has been collected on priority groups for an initial HIV vaccination by policy makers, although of a limited sample size. The ranking suggests the possibility of political and social confrontations in some countries where certain practices are not legalised or widely socially accepted. In other words, in countries where adult consensual same-sex relations or injecting drug use are widely criminalized, policy considerations might be unfairly prejudiced against such risk groups. The authors acknowledge the potential homophobic implications inherent in this observation and suggest the need for broad discussions to encourage evidence-based decisions on the distribution of a future HIV vaccine. Therefore, it is expedient in the light of delays in vaccine coverage experienced with other sexually transmitted disease (STD) vaccines e.g. Human Papilloma Virus (HPV) vaccine, that pre-emptive assessments of this kind are done on larger scales to facilitate early planning.

Participants in this study ranked vaccine efficacy, vaccine safety, vaccine cost and vaccine type in decreasing order of importance as potential factors that would influence an HIV vaccine's acceptability. 'Vaccine type' included elements such as number of doses, virus strain coverage and route of administration. In terms of vaccine cost, 1 out of 4 participants would be willing to pay 'any amount' 'out-of-pocket' for a vaccine with efficacy $\geq 95.98 \%$ and 1 out of 2 would pay up to 100 USD for a full course. The participants' responses to how much they would be willing to pay, reflect their personal opinion, and not that of the groups/countries they belong to. Similar results were found in a study of US adolescents where higher HIV vaccine acceptability was associated with greater vaccine efficacy and lower vaccine costs [14]. Furthermore, another study among highrisk communities in the US suggested that consumer concerns about low to moderate efficacy vaccines and the fear of side effects may limit the acceptability of an HIV vaccine [15]. Thus these limited studies suggest that characteristics of a future HIV vaccine would influence its acceptability, and that rapid, high vaccine coverage is not guaranteed. A more recent study used 'conjoint analysis', to assess multi-attribute factors in order to more closely approximate decisions about 
'real-world' product acceptability compared to the traditional single item factors, and identified vaccine efficacy as having the greatest impact on HIV vaccine acceptability [16]. According to that study, the 'theoretical best vaccine' with the following attributes: 95\% efficacy, lifetime protection, protection against multiple HIV subtypes, requiring 1 dose only, administered orally, with no side effects, and a cost of 10 USD would have an overall acceptability of $88.1 \%$ (S.D. =30). On the contrary, the study estimated the overall acceptability of the worst possible vaccine as $31.8 \%$ (S.D. $=38.4$ ) [16]. While some of the attributes of this 'theoretical best vaccine' may not even be feasible or practical (e.g. very high efficacy [such as the arbitrarily chosen value of $95.98 \%$ in our questionnaire], oral administration, lifetime protection, costing 10 USD), the emphasis lies on the low overall acceptability which highlights the critical need to identify and address the determinants of acceptability. Thus, the first generation HIV vaccine which may have low to moderate efficacy, in the order of $50 \%$ [11] should be expected to face the challenges of low acceptance rates and less optimal uptake. An estimated future global HIV vaccine uptake according to a WHO-UNAIDS panel of experts was $38 \%$ for a high efficacy vaccine (where 'high efficacy' is defined as VE>70\%) and $19 \%$ for a low to moderate efficacy vaccine (VE: 30-50\%) [17]. In addition, a systematic review and meta-analysis on future HIV vaccine acceptability in 2010 revealed a moderate level of HIV vaccine acceptability (65.6\%; S.D. = 21.1) across 20 studies (involving 7576 subjects), and identified vaccine attributes and HIV risk perception among the key correlates of acceptability [18].

The results also indicate that 2 out of 3 policy makers would be 'positively' predisposed to a vaccine that fails to achieve protection against infection $\left(\mathrm{VE}_{S} /[\mathrm{a}]\right)$ but attains the other measures of efficacy $[\mathrm{b}]$ and [c] (i.e. reduced rate of progression in vaccinated infected persons, and reduced infectiousness in vaccinated infected persons, respectively). According to 1 out of 3 policy makers, such a vaccine will be only acceptable if it has minimal cost to allow a wider distribution to their countries (or regions). About 1 out of 2 policy makers would be 'negatively' predisposed to this kind of vaccine being supported by their country's (or region's) health budget and roll-out. Furthermore, fifty-five percent of policy makers agree with targeting populations with higher risk of 
HIV infection where the candidate HIV vaccine has low efficacy. And a vaccine that is assessed to be not cost-effective would not be supported by $55 \%$ of policy makers till it achieves costeffectiveness measures. Policy makers would prefer to continue promoting existing combination prevention and other interventional strategies in the latter scenario. These results indicate that the acceptability of a future HIV-1 vaccine is not automatically guaranteed and is likely to be shaped by vaccine characteristics and opinion.

\section{Study Limitations}

This study is limited by its small convenience sample with a $24.3 \%$ response rate, and having responses from participants representing only 3 out of 6 WHO regions. Our results cannot therefore be generalised to the other WHO regions, as they may not reflect the views of policymakers in these regions. A much larger study needs to be done to provide more generalisable data. Furthermore, our small sample size also implies the study is only descriptive and precludes statistical testing of the data.

\section{Conclusions}

The quest for the elusive HIV-1 vaccine rages on. The challenges are grim; the promises few. Albeit, an effective HIV-1 vaccine delivered as part of a comprehensive HIV prevention package involving behavioral and health promotion strategies, holds the best promise at winning the war over the HIV pandemic.

Failed efficacy trials will provide some insight into the quest for an effective HIV-1 vaccine for use in better designed trials. The failed efficacy trials would also offer the opportunity to carefully evaluate unsuccessful vaccine design strategies. For instance, cell mediated immunity induced by vaccines would be expected to attempt to suppress viral replication, and would not be expected to prevent infection. Hence the observation that early plasma viral loads were unaffected in cell mediated vaccines trials calls for further inquests to clarify whether this failure was a result of the limited quantitative and qualitative profile of vaccine-induced cell mediated immune response or a 
more fundamental incapacity of cell-mediated immunity vaccines to alter the course of an established HIV infection.

Finally, the identification of personal behavioral and societal factors that may negatively impact the smooth transition of a successful HIV vaccine from the laboratory to the 'real world' need to be made and their solutions rehearsed in anticipation of an HIV vaccine. Existing national vaccine distribution infrastructure need to be strengthened to be able to take on an HIV vaccine programme without negative outcomes.

\section{Acknowledgements}

The authors would like to thank Drs Antoinette Ba-Nguz and Alex Adjagba for their help in reaching respondents from Africa. 


\section{Bibliography}

1. James M. Binley, T.W., Bette Korber, Michael B. Zwick, Meng Wang, Colombe Chappey, Gabriela Stiegler, Renate Kunert, Susan Zolla-Pazner, Hermann Katinger, Christos J. Petropoulos, and Dennis R. Burton, Comprehensive Cross-Clade Neutralization Analysis of a Panel of AntiHuman Immunodeficiency Virus Type 1 Monoclonal Antibodies. JOURNAL OF VIROLOGY, 2004. 78(23): p. 13232-13252.

2. Zhang PF, C.F., Dong M, Choudhary A, Bouma P, Zhang Z, et al, Extensively crossreactive anti-HIV-1 neutralizing antibodies induced by gp140 immunization. Proceedings of the National Academy of Sciences of the United States of America., 2007. 104(24): p. 10193-8. 3. Lianxing Liu, Y.H., Zhenwu Luo, Yang Huang, Xintao Hu, Ying Liu, and and Y. Shao, Broad HIV-1 Neutralizing Antibody Response Induced by Heterologous Gp140/Gp145 DNA Prime-Vaccinia Boost Immunization. Vaccine, 2012. 30(28): p. 4135-4143.

4. Izabela Bialuk, S.W., Vibeke Andresen, Ruth H. Florese, Janos Nacsa, Valentina Cecchinato, Valerio W. Valeri, Jean-Michel Heraud, Shari Gordon, Robyn Washington Parks, David C. Montefiori, David Venzon, Thorsten Demberg, Marjorie Robert Guroff, Gary Landucci, Donald N. Forthal, and Genoveffa Franchini, Vaccine Induced Antibodies to the First Variable Loop of Human Immunodeficiency Virus Type 1 gp120, Mediate Antibody-Dependent Virus Inhibition in Macaques. Vaccine, 2011. 30(1): p. 78-94.

5. HL, R., Non-neutralizing antibodies in prevention of HIV infection. Expert Opin Biol Ther., 2013. 13(2): p. 197-207.

6. Daniela Santoro Rosa, S.P.R., Rafael Ribeiro Almeida, Eliane Conti Mairena, Edilberto Posto, Jorge Kalil, Edecio Cunha-Neto, A DNA Vaccine Encoding Multiple HIV CD4 Epitopes Elicits Vigorous Polyfunctional, Long-Lived CD4+ and CD8+ T Cell Responses. Plos one, 2011. 6(2): p. e16921.

7. Supachai Rerks-Ngarm, P.P., Sorachai Nitayaphan, Jaranit Kaewkungwal, Joseph Chiu, Robert Paris, Nakorn Premsri, Chawetsan Namwat, Mark de Souza, Elizabeth Adams, Michael Benenson, Sanjay Gurunathan, Jim Tartaglia, John G. McNeil, Donald P. Francis, Donald Stablein, Deborah L. Birx, Supamit Chunsuttiwat, Chirasak Khamboonruang, Prasert Thongcharoen, Merlin L. Robb, Nelson L. Michael, Prayura Kunasol and Jerome H. Kim, for the MOPH-TAVEG Investigators, Vaccination with ALVAC and AIDSVAX to Prevent HIV-1 Infection in Thailand. NEJM, 2009. 361(23).

8. Bogard, E. and K.M. Kuntz, The impact of a partially effective HIV vaccine on a population of intravenous drug users in Bangkok, Thailand: a dynamic model. Journal of acquired immune deficiency syndromes (1999), 2002. 29: p. 132-141.

9. Andersson, K.M., A.D. Paltiel, and D.K. Owens, The potential impact of an HIV vaccine with rapidly waning protection on the epidemic in Southern Africa: Examining the RV144 trial results. Vaccine, 2011. 29: p. 6107-6112.

10. Fowkes FJ, S.J., Beeson JG., Implications of the licensure of a partially efficacious malaria vaccine on evaluating second-generation vaccines. BMC Med, 2013. 30(11): p. 232.

11. Report, W.-U., Future access to HIV vaccines. 2000. 15(October): p. W27-W44.

12. Newman, P.a., et al., Endorsement of compulsory HIV vaccination policy among populations at high risk of HIV exposure (LA VOICES). Prevention science : the official journal of the Society for Prevention Research, 2014. 15(3): p. 428-35.

13. Weinbaum, C., K. Billah, and E.E. Mast, HEpatitis b vaccination coverage among adultsunited states, 2004. JAMA, 2006. 296(1): p. 33-34.

14. Webb, P.M., et al., HIV immunization: acceptability and anticipated effects on sexual behavior among adolescents. The Journal of adolescent health : official publication of the Society for Adolescent Medicine, 1999. 25(5): p. 320-2.

15. Newman, P.A., et al., Posttrial HIV vaccine adoption: concerns, motivators, and intentions among persons at risk for HIV. Journal of acquired immune deficiency syndromes (1999), 2004. 37: p. 1393-1403. 
16. Newman, P.A., et al., HIV vaccine acceptability among communities at risk: The impact of vaccine characteristics. Vaccine, 2006. 24: p. 2094-2101.

17. Esparza, J., et al., Estimation of "needs" and "probable uptake" for HIV/AIDS preventive vaccines based on possible policies and likely acceptance (a WHO/UNAIDS/IAVI study). Vaccine, 2003. 21(17-18): p. 2041-2050.

18. Newman, P.A. and C. Logie, HIV vaccine acceptability: a systematic review and metaanalysis. 2010. p. 1749-1756. 
Table 1: HIV vaccine strategy and willingness to pay

Factor, $\mathrm{N}=9$

Number Percentage

Preferred strategy for an INITIAL HIV immunization programme

To vaccinate Everyone at risk

6

44.4

To vaccinate Individuals at highest risk

1

11.1

To vaccinate Targeted risk groups

7

44.4

Amount willing to pay for HIV vaccine with $\geq 95.98 \%$ efficacy and

lifelong protection(Out of pocket)

Any amount

2

25.0

Up to US\$1000 for a course

Up to US\$500 for a course

1

12.5

Up to US\$100 for a course

4

50.0

Table 2: Vaccine characteristics and their acceptability

Attributes that will influence the accepta-

bility of an HIV vaccine the most

Rank(highest priority (=1) to least priority

$(=4)$ )

High Vaccine Efficacy

6(66.7)

2(22.2) 1(11.1)

High Vaccine Safety

$3(33.3)$

$3(33.3) \quad 2(22.2)$

$1(11.1)$

Vaccine type (number of doses required;

route; virus strain coverage)

$1(11.1) \quad 2(22.2) \quad 6(66.7)$

Vaccine Cost

$3(33.3) \quad 4(44.4) \quad 2(22.2)$ 
Table 3: Vaccine efficacy (VE) characteristics and their acceptability $[a]=V E_{S} ;[b]=V E_{P} ;$ and $[c]=V E_{I}$

\begin{tabular}{|c|c|c|c|}
\hline \multirow[t]{2}{*}{ Statement } & \multicolumn{3}{|c|}{ Responses } \\
\hline & 'positive' & '0' & 'negative' \\
\hline $\begin{array}{l}\text { A vaccine that has no VEs but VEp plus } \\
\text { VEi will be acceptable to me }\end{array}$ & $6(66 \%)$ & $1(11 \%)$ & $2(22 \%)$ \\
\hline $\begin{array}{l}\text { A vaccine that has no VEs but VEp plus } \\
\text { VEi will only be acceptable if it has very } \\
\text { minimal cost to allow universal vaccina- } \\
\text { tion of my country (or region) }\end{array}$ & $3(33 \%)$ & $2(22 \%)$ & $4(44 \%)$ \\
\hline $\begin{array}{l}\text { A vaccine that has no VEs but VEp plus } \\
\text { VEi will still be prioritized in my country's } \\
\text { health budget for promotion and roll-out } \\
\text { even though it may not be attractive to re- } \\
\text { cipients* }\end{array}$ & $3(38 \%)$ & $1(13 \%)$ & $4(51 \%)$ \\
\hline $\begin{array}{l}\text { A vaccine with low efficacy may be target- } \\
\text { ed only to populations with higher risk of } \\
\text { HIV infection, especially if those popula- } \\
\text { tions are not easily reachable with other } \\
\text { preventive interventions. }\end{array}$ & $5(55 \%)$ & $0(0 \%)$ & $4(44 \%)$ \\
\hline $\begin{array}{l}\text { A vaccine that is not cost-effective in the } \\
\text { light of my country's economy will not be } \\
\text { acceptable until it becomes more cost- } \\
\text { effective. I would rather recommend in- } \\
\text { vesting in promoting existing interventions }\end{array}$ & $5(55 \%)$ & $3(33 \%)$ & $1(11 \%)$ \\
\hline
\end{tabular}


Please refer to text for definitions. 'Positive' = responses ' 1 ' and ' 2 ', ' 0 ' means 'neutral', and 'negative' $=$ responses ' 4 ' and ' 5 ' on the Likert scale. * The percentages for this question differ from those of the other questions in this table, as one participant did not respond. 